\title{
Classification of Water Stress in Sunagoke Moss Using Color Texture and Neural Networks
}

\author{
Stephen N. ONDIMU and Haruhiko MuRAse \\ Bioinstrumentation, Control and Systems (BICS) Engineering Laboratory, \\ School of Life and Environmental Sciences, Osaka Prefecture University, \\ 1-1 Gakuen-cho, Sakai, Osaka 599-8531, Japan
}

(Received February 24, 2008)

\begin{abstract}
The general appearance of a plant is the most obvious indicator of its physiological wellbeing. Color Co-occurrence Matrix (CCM) texture features, extracted from a set of 1095 images were used to classify water stress in Sunagoke moss (Rhacomitrium canescens) using Neural Networks (NN). An Excess Green Water Stress Index (EGWSI) was developed and used to quantify water stress in the sample. The CCM texture features were extracted from: red-greenblue (RGB); hue-saturation-intensity (HSI) and CIE's (Comission Internationale de LEclairage) LAB and XYZ color spaces. The HSI texture features achieved $99.45 \%$ water stress classification efficiency. They were followed by RGB, XYZ and LAB texture features with classification efficiencies of $99.07 \%, 98.83$ and $96.3 \%$ in that order respectfully. The HSI textures features displayed a higher ability and reliability to classify water stress in Sunagoke moss and can be used for stress detection under varying light intensities. A significant accomplishment of this study was the detection of both flood and draught water stress in a plant that exhibits a high level of desiccation tolerance. This provides an opportunity for the possibility of allowing plants to control their own bioproduction environments.
\end{abstract}

Keywords: Color Co-occurrence Matrix (CCM), color space, Neural Networks (NN), StressImaging, physiological well-being

\section{INTRODUCTION}

Due to a number of good qualities, Sunagoke moss (Rhacomitrium canescens) has attracted much interest as a good potential material for bio-wall and bio-roof greening for Urban Heat Island (UHI) mitigation (Ondimu and Murase, 2007; Ondimu and Murase, 2006; Murase and Ushada, 2006). This plant is: resilient with minimum maintenance; environmentally sound with no known disease or pest; requires no soil growing media and retains its dark green color even in temperatures as low as $-30^{\circ} \mathrm{C}$ or as high as $40^{\circ} \mathrm{C}$. In addition, it exhibits a high level of desiccation tolerance (Aro et al., 1981; Bowen, 1933; Valanne, 1984) making it an ideal plant for studying the robustness of water stress detection techniques in plants. Like other mosses, its optimum rates of net fixation are achieved only at certain water contents, below which it is incapable of photosynthesis and above which photosynthetic activity declines (Oechel and Collins, 1976; Proctor, 1980). Thus, water stress is the main factor limiting its production (Skre and Oechel, 1981). To establish water contents favoring its optimum rates of net fixation, water states triggering its deficit and excess

Corresponding author: Stephen N. Ondimu, fax : +81-72-254-9918, e-mail : Ondimu@bics.envi.osakafu-u.ac.jp 
water stresses need to be determined. It is believed that establishment of optimum points of net fixation will lead to optimum productivity (quality and quantity) of Sunagoke moss to meet its anticipated demand.

\section{Plant water stress detection by visual imaging}

The general appearance of plants is the most obvious indicator of their physiological wellbeing. However, by the time stress symptoms become apparent to humans, the plant may be already severely affected. Water stress in plants can be caused by too much water (excess water stress) or too little water (deficit water stress). It can be expressed at DNA, cellular, organ, organism and ecosystem levels. Water stress causes changes in patterns of leaf cooling (due to active regulation of stomata aperture), changes in internal and surface leaf structure and leads to breakdown of photosynthetic pigments. These changes can be detected by imaging. Imaging techniques make pre-symptomatic detection of physiological changes in plants possible and in real-time. Some of the imaging techniques applied in plants include fluorescence imaging, bioluminescence imaging, thermal imaging, magnetic resonance imaging and reflectance imaging (Chaerle et al., 2001).

Visual imaging of plant canopies provides indirect data which can be used to derive their structural or functional changes indicating stress (Peñuelas and Filella, 1998). This technique has been widely used in detecting and quantifying physiological changes and accompanying biotic and abiotic stressors in plants (Kacira et al., 2002; Carter and Miller, 1994; Kacira and Ling, 2001; Foucher et al., 2004; Ceccato et al., 2001; Mirik et al., 2006; Díaz-Lago et al., 2003). Most of these investigations used either gray-level textural features or color information features based on the Red-Green-Blue (RGB) model. However, Matas et al. (1995) observed that colors in digital images depend on: characteristics of the illumination source, spectral reflectance of objects, position of the illumination and position of the camera. Thus, non-normalized RGB coordinates could be greatly varied under such conditions and simple direct extraction of features from such images may lead to erroneous interpretations.

Levine (1985) observed that textures represent the phenomenon of shift invariance in image structure where perception of image is not affected by position within the image. Statistical texture features are random and often occur naturally. Julez (1962) introduced the spatial gray-level dependencies method for statistical texture analysis. Haralick and Shanmugan (1973) generalized the concept of using second order statistics into special gray-level dependence matrices (SGDM) in the gray-level co-occurrence matrix (GLCM) method. Later, Haralick and Shanmugan (1974) used these SGDMs to develop an associated set of 16 textural features. Though the GLCM texture has been widely used, relatively little is known about the relationship between color information and textural attributes (Yin and Panigrahi, 2004). In addition, grey-level texture features tend to be globally adaptable but are not locally optimized.

Shearer (1986) suggested use of color texture analysis to overcome the shortcomings of traditional grey-level texture analysis. Shearer and Holmes (1990) reported 91\% accuracy in classifying different types of nursery stock by color co-occurrence matrices (CCM) texture. Burks et al. (2000) reported 93\% accuracy in classifying six weed species using the CCM texture and discriminant analysis. In a related study, Pydipati et al. (2006) reported 95\% accuracy in identifying citrus disease using CCM texture features. Meyer et al. (1998) used RGB true color to generate an excess green feature to discriminate between four species of weeds and soil with $99 \%$ accuracy. In another study, Sena et al. (2003) used normalized excess green index based texture analysis to identify armyworm-damaged maize plants. This paper reports further contribution towards enhancing these novel techniques. In the study, CCM texture and neural networks were used to classify water stress in Sunagoke moss. 


\section{Color co-occurrence matrix method}

The CCM texture analysis method is based on the hypothesis that use of color features in the visible spectrum provides additional image characteristics over the traditional gray-level representation. The method consists of: 1) transforming RGB images from RGB color space to a color space familiar to humans; 2) generation of SGDMs and 3) determination of Haralick textural features. The SGDMs measure the probability that a pixel at a particular gray-level will occur at a distinct distance and orientation from any pixel given that pixel has a second particular gray-level (Shearer and Holmes, 1990). SGDMs are represented by the function P (a, b, d, $\theta$ ) where a, represents the gray-level at location $(\mathrm{x}, \mathrm{y})$ in image $\mathrm{I}(\mathrm{x}, \mathrm{y})$, and $\mathrm{b}$ represents the gray-level of pixel at distance $\mathrm{d}$, from location (x, $\mathrm{y}$ ) at an orientation angle of $\theta$ (Shearer, 1986). Figure 1 shows an illustration of a gray-level image and its SDGM at $\theta=0^{\circ}$ and $\mathrm{d}=1$. Figure 1(a) shows the reference mask where the reference pixel $\mathrm{P}(\mathrm{x}, \mathrm{y})$ is shown in asterisk. All the eight neighbors shown are at distance $\mathrm{d}=1$, from the reference pixel. By symmetry neighbors at position one and five are considered to be at orientation $\theta=0^{\circ}$, while positions four and eight are considered to be at $\theta=45^{\circ}$. Any given texture feature $\mathrm{x}$ is computed from the SGDM using the relation in equation 1.

In the study, RGB images were transformed into three color spaces: hue, saturation and intensity (HSI), and CIE's (Comission Internationale de LEclairage) LAB and XYZ. Each pixel map in each color space was used to generate one CCM resulting in three co-occurrence matrices for each color space. Texture features were extracted at one pixel offset distance and $0^{\circ}, 45^{\circ}, 90^{\circ}$ and $135^{\circ}$ orientations. Features from the four orientations were then averaged before being used to develop neural network (NN) classifiers for water stress in the Sunagoke moss sample.

$$
\mathrm{x}=\frac{\sum_{\mathrm{a}=0}^{\mathrm{N}_{\mathrm{s}}-1} \sum_{\mathrm{b}=0}^{\mathrm{N}_{\mathrm{s}}-1} \mathrm{~W}(\mathrm{a}, \mathrm{b}) \mathrm{p}(\mathrm{a}, \mathrm{b}, \mathrm{d}, \theta)}{\mathrm{N}}
$$

where: $\mathrm{N}_{\mathrm{g}}$ is the total gray-level, $\mathrm{W}(\mathrm{a}, \mathrm{b})$ the weighting on of function used to generate the feature in question and $\mathrm{N}$ is the total number of pixels in the image.

Goal and objectives

The overall goal of this research is to develop a StressImaging system for water stress detection and diagnosis in a closed bioproduction system for cultured Sunagoke moss. The objectives were to:

- Generate CCM texture features using images of Sunagoke moss under different daily average water states.

- Train, test and validate NN models to classify water stress in Sunagoke moss using features generated in the previous objective.

- Determine which of the CCM textural features extracted from RGB, HSI, LAB and XYZ color spaces have a higher water detection.

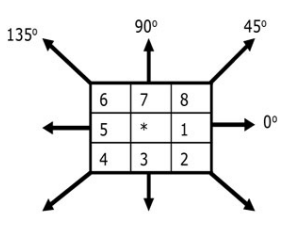

(a)

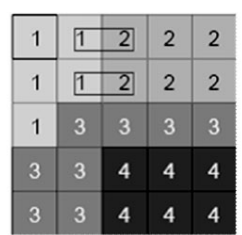

(b)

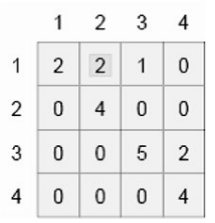

(c)

Fig. 1 Gray-level dependence illustration (a) nearest neighbor diagram, (b) gray-level image and (c) SGDM matrix for orientation $0^{\circ}$ at pixel distance $=1$. 


\section{MATERIALS AND METHODS}

\section{Sunagoke moss sample}

Figure 2 shows the Sunagoke moss sample and a production system in which the StressImaging system being developed in this study is to be implemented. The production system (Fig. 2(a)) is meant to produce tile like living units called Sunagoke moss mats (Fig. 2(b)) engineered for ease of installation as cladding on non-horizontal surfaces. The units are made of living cultured Sunagoke moss growing in polyvinyl chloride (PVC) netting and anchored in glass wool media. A sample of cultured Sunagoke moss (Fig. 2(c)), growing on cotton wool media and placed in a $100 \mathrm{~mm} \times 75 \mathrm{~mm} \times 25 \mathrm{~mm}$ glass vessel was used in this study. Prior to commencement of image acquisition, pre-determined amount of water (based on dry weight) was applied to the sample. Three different data sets were obtained corresponding to: $4 \mathrm{gg}^{-1}$; (grams of water per gram of dry Sunagoke moss), $2 \mathrm{gg}^{-1}$ and $1.5 \mathrm{gg}^{-1}$ initial water contents of the sample. At the start of each day of the experiment, the water state of the sample was computed in $\mathrm{gg}^{-1}$.

Experimental data acquisition system

Figure 3 is a summary of the procedure used to classify water stress in this study. RGB images of size $480 \times 640$ pixels were acquired with a CCD video camera, TRV22E (Sony cooperation, Japan) placed at $450 \mathrm{~mm}$ perpendicular to the sample surface as shown in Fig. 4. Images were acquired after every $10 \mathrm{~min}$ (73 images per day) for five consecutive days. A total of 1095 images were acquired. Light was provided by two 22W lamps (EFD25EN/22, National Corporation, Japan). Data acquisition was conducted in a walk-in growth chamber (NK-system) with temperature and humidity set to $15^{\circ} \mathrm{C}$ and $60 \%$ respectively. The light: dark period was $12 \mathrm{~h}$. After acquisition, ImageJ (Rasband, NIH) software was used for processing and analyzing the images.

\section{Water stress classification}

Excess green water stress index

After acquisition, the images were pre-processed by pixel size reduction. An excess green water stress index (EGWSI) was determined using equation 2 and used to quantify water stress in the sample.

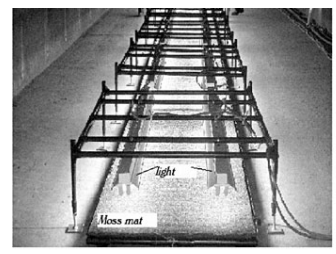

(a)

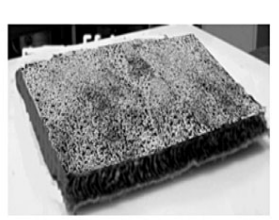

(b)

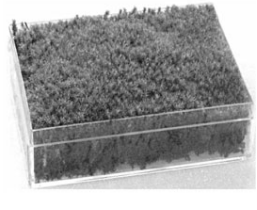

(c)

Fig. 2 Sunagoke moss: (a) production system (b) mat (c) sample used in this study.

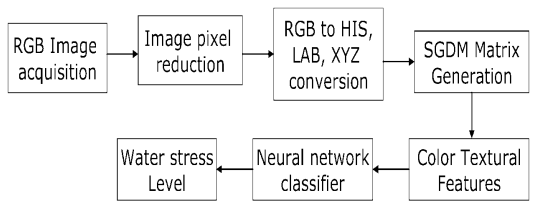

Fig. 3 Water stress detection process. 


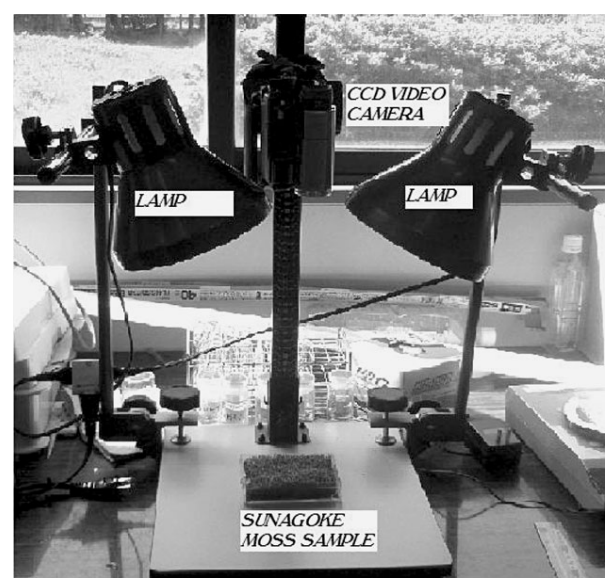

Fig. 4 Image acquisition system.

$$
\begin{gathered}
\text { EGWSI }=\frac{A G_{\mathrm{E}} I-A G_{\mathrm{E}} \mathrm{I}_{\mathrm{U}}}{A \mathrm{AG}_{\mathrm{E}} \mathrm{I}_{\mathrm{L}}-A \mathrm{AG}_{\mathrm{E}} \mathrm{I}_{\mathrm{U}}} \\
\mathrm{AG}_{\mathrm{E}} \mathrm{I}=\frac{2 \mathrm{G}-\mathrm{R}-\mathrm{B}}{\mathrm{N}}
\end{gathered}
$$

where: NGEI = average excess green index given by equation 3; $\mathrm{AG}_{\mathrm{E}} \mathrm{I}_{\mathrm{U}}=$ average excess green index of a non-respiring plant (upper limit); $\mathrm{AG}_{\mathrm{E}} \mathrm{I}_{\mathrm{L}}=$ average excess green index of a non-stressed plant (lower limit); R, G and B are the pixel intensity values for the red, green and blue channels and $\mathrm{N}$ is as defined in equation 1.

Figure 5 shows the variation of EGWSI values with time for the sample at initial water states of $4 \mathrm{gg}^{-1}, 2 \mathrm{gg}^{-1}$ and $1.5 \mathrm{gg}^{-1}$. Using these results and the daily average water states of the sample, its water stress was classified into three levels: level 1 (no water stress), level 2 (excess water stress) and level 3 (deficit water stress).

\section{Textural feature extraction}

The pre-processed images were converted to HSI, LAB and XYZ color spaces. For every band in each color space, SGDMs were generated. Ten Haralick (Haralick et al, 1973) textural features: second moment, mean, variance, covariance, correlation, inverse difference moment, maximum probability, contrast, entropy and product moment were extracted from the SGDMs giving 30 features for each image.

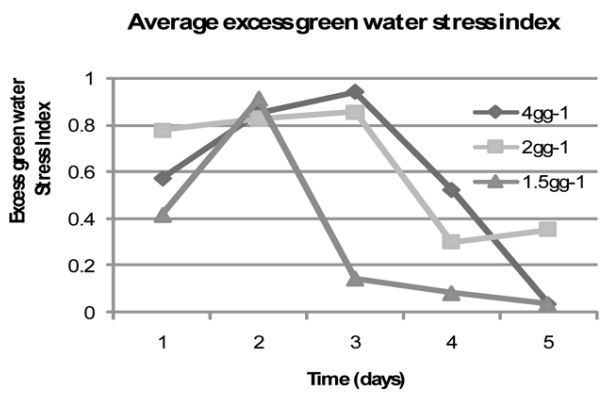

Fig. 5 Variation of excess green water stress index with time. 
A total of 1095 data patterns comprising of CCM textural features and corresponding water stress levels were formulated.

Neural network classifiers

Four NN classifiers: C1, C2, C3 and C4 corresponding to RGB, HSI, LAB and XYZ color textures, respectively were developed for water stress classification in the sample. A general architecture of 30:6:1 (input: hidden: output) was selected by trial and error and optimized using the RGB texture features. It was then adapted for all the classifiers. Of the input-output data patterns, $25 \%$ were selected at random and used for validation while the remaining $75 \%$ were used for training the classifiers. A learning rate of 0.5 and momentum of 0.3 were used. Five trial models were trained in 300 iterations and validated for each classifier. At the start of every trial, weights were randomly initialized. Average misclassification error i.e. percent of records in the training/validation data, the classifier predicted the class wrongly was used to evaluate the performance of the classifiers.

\section{RESULTS}

Figure 6 shows average training and validation misclassification errors of the four NN classifiers when using 10 textural features. The error bars shown indicate the standard deviation of five trials for each classifier. All the classifiers exhibited negligible training misclassification error. Classifier $\mathrm{C} 2$ exhibited the lowest misclassification error followed by $\mathrm{C} 1, \mathrm{C} 4$ and $\mathrm{C} 5$ in that order. From these results, it can be deduced that HSI color texture features as espoused in classifier $\mathrm{C} 2$ have the highest water stress detection ability.

When the texture features were reduced to five (energy, homogeneity, contrast, mean and maximum probability) the misclassification errors of the four classifiers were as shown in Fig. 7. After reduction to three features (energy, homogeneity and contrast) the misclassification errors were as shown in Fig. 8. The error bars in both figures indicate the standard deviations of five trials for each classifier.

Figure 9 is a comparison in water stress misclassification error when texture features extracted from combination of 2 color channels of the HSI and RGB color spaces. This comparison was driven by the fact that RGB is the color space at which images were acquired in this study, while HSI is the color space closest to the way humans perceive color.

\section{DISCUSSION}

In this study, a set of 1095 images of a Sunagoke moss sample at initial water states of: 4.0 $\mathrm{gg}^{-1} ; 2 \mathrm{gg}^{-1}$ and $1.5 \mathrm{gg}^{-1}$ were used to classify water stress in the sample. The HSI texture features

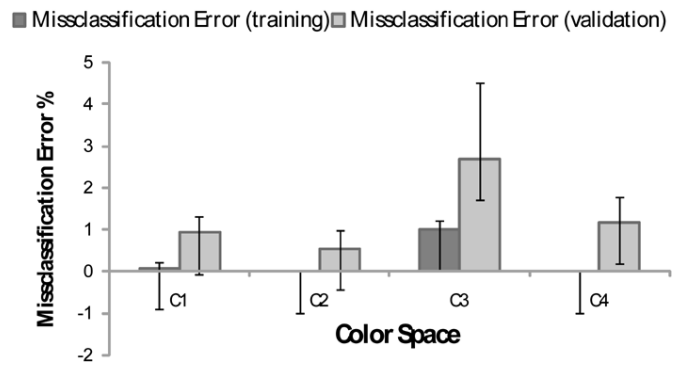

Fig. 6 Training and validation misclassification errors when using10 texture features: second moment, mean, variance, covariance, correlation, inverse difference moment, maximum probability, contrast, entropy and product moment. 
PHYSIOLOGICAL STRESS IMAGING

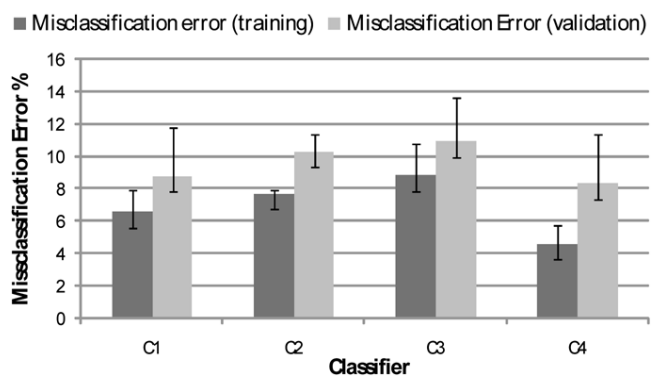

Fig. 7 Training and validation misclassification errors when using five textural features: energy, homogeneity, contrast, mean and maximum probability.

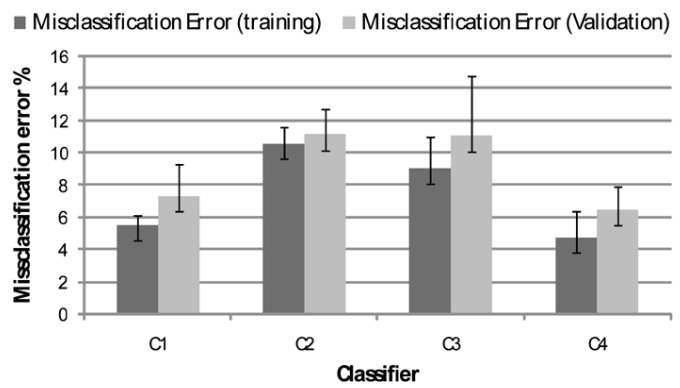

Fig. 8 Training and validation misclassification errors when using three texture features: energy, homogeneity and contrast.

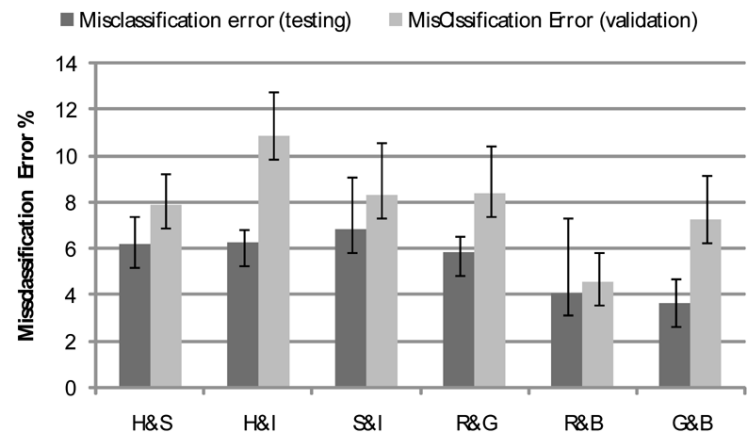

Fig. 9 Training and validation misclassification errors for HSI and RGB color spaces texture features.

showed $99.45 \%$ classification accuracy when 10 textural features (second moment, mean, variance, covariance, correlation, inverse difference moment, maximum probability, contrast, entropy and product moment) were used. They were followed by RGB (99.05\%), XYZ (98.83\%), and LAB $(96.3 \%)$ texture features in that order, respectfully. When the features were reduced to five (energy, homogeneity, contrast, mean and maximum probability) the classification accuracy was $91.17 \%, 89.67 \%, 91.63 \%$ and $89.02 \%$ for RGB HIS, XYZ and LAB texture features respectively. On the hand, the classification efficiency was $92.61 \%, 88.81 \%, 93.68 \%$ and $88.92 \%$ for RGB, HSI, $\mathrm{XYZ}$ and LAB texture features respectfully, when the number of features used was reduced to three: energy, homogeneity, contrast.

In general, the results in Figs. 6, 7 and 8 depict increasing misclassification error with 
decreasing number of textural features. This was especially most pronounced in the HSI color space. However, this could be unique for the nature of texture features selected. Classifiers $\mathrm{C} 1$, and $\mathrm{C} 4$ based on the RGB and XYZ color texture performed better than classifier $\mathrm{C} 2$ when using five and three textural features. However, classifier $\mathrm{C} 2$ exhibited a lower standard deviation in both cases. This implies that HSI color features are more reliable in classifying water stress. This can be attributed to the fact that the HSI color space is closest to the way the human vision system perceives color. The performance of classifiers $\mathrm{C} 1$ and $\mathrm{C} 4$ (based on RGB and XYZ color texture respectfully) is comparable. This can be attributed to the fact that the RGB and XYZ color models are machine based color spaces: RGB color space being the model at which image acquisition is done and XYZ model being the color space at which images are displayed on screens.

From Fig. 9, hue-saturation based texture displayed the lowest water stress misclassification error in the sample for the HSI color space. This implies that by discarding the intensity component, texture features extracted from the HSI model can be used for stress detection under varied lighting conditions with a reasonable degree of accuracy. This is an important characteristic as it makes it possible to use imaging for stress detection under natural growth environments. Texture features extracted from red-blue channels displayed the lowest misclassification error for the RGB color space. This can be attributed to the fact that plants absorb light photons with $435-480 \mathrm{~nm}$ (blue) wavelengths and 650-675 nm (red) wavelengths (Cotterill, 2002) and they reflect the photons less than those with wavelengths outside these ranges. Increase in absorption in the red and blue wavelengths leads to decrease in reflection in the same wavelengths and vice versa. An increase in absorption indicates higher physiological well-being and less stress. On the other hand decrease in absorption implies lower physiological well-being and higher stress. Thus changes in pixel intensities of the red and blue channels of RGB images are better indicators of physiological stress levels in plants.

In conclusion, HSI CCM texture features have a higher ability and reliability to detect water stress both in real-time and presymptomatically, in Sunagoke moss than those extracted from RGB, $\mathrm{XYZ}$ and LAB color spaces. The CCM texture analysis method can be used for detection of: 1) both drought and flood water stress and 2) water stress under varying lighting conditions in Sunagoke moss. Though, Sunagoke moss was used in this study, this method can be extended to other plants. Use of hyperspectral reflectance imaging can enable this method to detect specific stress signatures for particular plants and make it a powerful biophysical, non-invasive and presymptomatic stress diagnosis method for a wide variety of stresses in plants. This will provide an opportunity for the possibility of allowing plants to control their own bioproduction environments.

\section{REFERENCES}

Aro, E. M., Niemi, H., Valanne, N. 1981. Structural and functional studies on bryophyte photonsythesis. In "Photonsythesis" (ed. by Akoyunoglou, G.), Vol. 3. Int. Sci. Serv., Balaban Pennesylvannia, p 327-335.

Bowen, E. J. 1933. The mechanism of water conduction in Musci considered in relation to habitat. II. Mosses growing in dry environments. Ann. Bot. 47: 889-912.

Burks, T. F., Shearer, S. A., Payne, F. A. 2000. Classification of weed species using color texture features and discriminant analysis. Trans. ASAE 43: 441-448.

Carter, A. G., Miller, L. R. 1994. Early detection of plant stress by digital imaging within narrow stresssensitive wavebands. Remote Sens. Environ. 50: 295-302.

Ceccato, P., Flasse, S., Tarantola, S., Jacquemound, S., Gregoire, J. 2001. Detecting vegetation water content using reflectance in the optical domain. Remote Sens. Environ. 77: 22-33.

Chaerle, L., Van Der Straeten, D. 2001. Seeing is believing: imaging techniques to monitor plant health. Biochim. Biophys. Acta 1519: 153-166. 


\section{PHYSIOLOGICAL STRESS IMAGING}

Cotterill, R. M. J. 2002. Biophysics; An introduction. John Wiley \& Sons, London, pp 395.

Díaz-Lago, J. E., Stuthman, D. D., Leornard, K. J. 2003. Evaluation of components of partial resistance to oat crown using digital image analysis. Plant Dis. 87: 667-674.

Foucher, P., Revollo, P., Vigouroux, B., Chassériaux, G. 2004. Morphological image analysis for the detection of water stress in potted Forsythia. Biosystems Engineering 89: 131-138.

Haralick, R. M., Shanmugan, K. 1974. Combined spectral and spatial processing of ERTS imagery data. Remote Sens. Environ. 3: 3-13.

Haralick, R. M., Shanmugan, K., Dinstein, I. 1973. Textural features for image classification. IEEE Transactions on Systems, Man and Cybernetics: SMC 3: 610-621.

Haralick, R. M., Shanmugan, K. 1973. Computer classification of reservoir sandstone. IEEE Trans. Geosci. Electr. GE 11: 171-177.

Julez, B. 1962. Visual pattern discrimination. IRE Trans. Info. Theory 8: 84-92.

Kacira, M., Ling, P., Short, T. H. 2002. Machine vision extracted plant movement for early detection of plant water stress. Trans. ASAE 45: 1147-1153.

Kacira, M., Ling, P. 2001. Design and development of an automated and Non-contact sensing system for continuous monitoring of plant health and growth. Trans. ASAE 44: 989-996.

Levine, B. 1985. Vision in man and machine. McGraw-Hill Book Co., New York, N.Y., pp 574.

Matas, J. R., Marik, R., Kitter, J. 1995. Colour-based object recognition under spectrally non-uniform illumination. Image and Vision Computing 13: 663-669.

Meyer, G. E., Mehta, T., Kocher, M. F., Mortensen, D. A., Samal, A. 1998. Textural imaging and discriminant analysis for distinguishing weeds for spot spraying. Trans. ASAE 41: 1189-1197.

Mirik, M., Michels, J. Jr., G. J., Kassymzhanova-Miril, S., Ellio, N. C., Catana, V., Jones, D. B., Bowling, R. 2006. Using digital image analysis and spectral reflectance data to quantify damage by Greenburg (hemitera: Aphididae) in winter and wheat. Comput. Electron. Agric. 51: 86-98.

Murase, H., Ushada, M. 2006. Machine vision applications for micro-precision agriculture. Environ. Control Biol. 44: 199-206.

Oechel, W. C., Collins, N. J. 1976. Comparative $\mathrm{CO}_{2}$ exchange in mosses from two tundra habitats at Barrow, Alaska, Canadian. Journal of Botany 54: 1355-1369.

Ondimu, S., Murase, H. 2006. Thermal properties of living roof greening material by inverse modeling. Applied Engineering in Agriculture 22: 435-441.

Ondimu, S. N., Murase, H. 2007. Combining Galerkin methods and neural network analysis to inversely determine thermal conductivity of living green roof materials. Biosystems Engineering 96: 541-550.

Peñuelas, J., Filella, I. 1998. Visible and near-infrared reflectance techniques for diagnosing plant physiological status. Trends in Plant Science 3: 151-156.

Proctor, M. C. F. 1980. Diffussion resistances in bryophytes. In "Plants and Their Atmospheric Environment” (ed. by Grace, J., Ford, E. D., Jarvis, P. G.), Blackwell, Oxford, UK, p 219-229.

Pydipati, R., Burks, T. T., Lee, W. S. 2006. Identification of citrus disease using color texture features and discriminate analysis. Comput. Electron. Agric. 52: 49-59.

Rasband, W. 2007. ImageJ 1.38r. National Institute of Health (NIH) USA. Available at: http://rsb.info.hih.gov/ij/

Sena, D. G., Jr., Pinto, F. A. C., Queiroz, D. M., Viana, P. A. 2003. Fall armyworm damaged maize plant identification using digital images. Biosystems Engineering 85: 449-454.

Shearer, S. A. 1986. Plant identification using color co-occurrence matrices derived from digitized images. Ph.D. Thesis, Ohio State University, Columbus, OH.

Shearer, S. A., Holmes, R. G. 1990. Plant identification using color co-occurrence matrices. Trans. ASAE 33: $2037-2044$.

Skre, O., Oechel, W. C. 1981. Moss functioning in different taiga ecosystems in interior Alaska 1. Seasonal, phenotypic and drought ettects on photosythesis and response patterns. Oecologia 48: 50-59.

Valanne, N. 1984. Photosynthesis and photosynthetic products in mosses. In "The Experimental Biology of Bryophytes” (ed. by Dyer, A. J., Duckett, J. G.), Academic press, London, p 257-273.

Yin, H., Panigrahi, S. 2004. Image processing techniques for internal texture evaluation of French Fries. Applied Engineering in Agriculture 20: 803-811. 